\title{
Mechanism of Side Chain Controlled Proton Conductivity in Bioinspired Peptidic Nanostructures
}

Subhasish Roy, ${ }^{\neq, f,+}$ Lianjun Zheng, ${ }^{,,+}$Ohad Silberbush, ${ }^{\ddagger}$ Maor Engel, ${ }^{*}$ Yoav Atsmon-Raz,, Yifat Miller, ${ }^{,, \pi}$ Agostino Migliore, ${ }^{*, \xi, \&}$ David N. Beratan, ${ }^{*, \xi, l l, \neq}$ and Nurit Ashkenasy ${ }^{*, \neq, \eta}$

¥ Department of Materials Engineering, \$ Department of Chemistry, and " Ilse Katz Institute for Nanoscale Science and Technology, Ben-Gurion University of the Negev P.O. Box 653, Beer-Sheva 84105, Israel

$\S$ Department of Chemistry and " Department of Physics, Duke University, Durham, North Carolina, 27708, United States

\& Department of Chemical Sciences, University of Padova, Via Marzolo, 1, 35131 Padova, Italy

‡ Department of Biochemistry, Duke University, Durham, North Carolina, 27710, United States

*email: Agostino Migliore: agostino.migliore@unipd.it, agostino.migliore@duke.edu; David Beratan david.beratan@duke.edu; Nurit Ashkenasy: nurita@bgu.ac.il

\section{Contents}

S1.Experimental methods

S1.1. Materials S2

S1.2. Solid-phase peptide synthesis and assembly $\quad$ S2

S1.3. Atomic force microscopy (AFM) S3

S1.4. Structural studies S3

S1.5. Electrical characterization of the peptide films S3

S1.6. Water uptake S4

S2. Additional experimental characterizations data S5

S3. Molecular Dynamics (MD) simulations

$\begin{array}{lll}\text { S3.1 Peptide systems } & \text { S7 }\end{array}$

S3.2 MD simulations $\quad \mathrm{S} 8$

S3.3 MD analysis $\quad$ S10

S4. Theoretical modeling

S4.1 Proton couplings and transfer rates S13

$\begin{array}{lll}\text { S4.2 Proton-transport model } & \text { S14 }\end{array}$

$\begin{array}{ll}\text { S5. Kinetic analysis of the MD snapshots } & \text { S17 }\end{array}$

$\begin{array}{lr}\text { S6. References } & \text { S20 }\end{array}$ 


\section{S1 Experimental methods}

\section{S1.1 Materials}

Fmoc-amino acids, 2-chlorotrityl chloride resin, coupling reagents, DIEPA, piperidine, acetic acid, trifluoroacetic acid (TFA), triisopropyl silane (TIS) were purchased from Novabiochem and Alfa Aesar. Peptide grade DMF was purchased from Biotech. The water used was triply distilled (TDW, EASYpure ${ }^{\circledR}$ RoDi, Thermo Scientific, USA, resistivity of $18.2 \mathrm{M} \Omega$ ). HPLC grade acetonitrile (JT Baker analyzed) was used. Other chemicals and reagents were purchased from Aldrich and Merck and were used without further purification unless otherwise specified.

\section{S1.2 Solid-phase peptide synthesis and assembly}

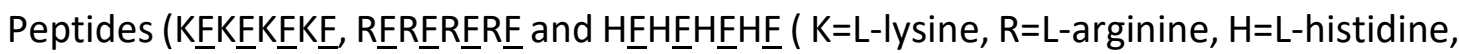
F=D-phenylalanine)) were synthesized in $0.2 \mathrm{mmol}$ scale on 2-chlorotrytyl chloride resin following routine Fmoc-solid phase synthesis protocols. ${ }^{1}$ Peptides cleavage from the resin was performed in a mixture of acetic acid (AcOH)/trifluoroethanol (TFE)/dichloromethane (DCM) having 1:1:3 ratio for $2 \mathrm{~h}$ at room temperature, followed by washing in DCM using a syringe filter for at least three times. The DCM solution of the side-chain protected peptides was diluted in hexane (1:3 mixture) and dried using a rotary evaporator. The side chain protected octa-peptides (25 $\mu \mathrm{mol})$ were cyclized in dry DMF (100 mL) using PyBOP $(39.0 \mathrm{mg}, 75 \mu \mathrm{mol})$ as a coupling reagent and DIEPA (N,N-Diisopropylethylamine, $26.1 \mu \mathrm{L}, 150 \mu \mathrm{mol}$ ) as a base. The cyclic peptide compounds were side-chain deprotected in a mixture of TFA/TIS/TDW with 95:2.5:2.5 ratio for $2 \mathrm{~h}$ at room temperature. The TFA solution was evaporated using air flow and the peptides were precipitated using diethyl ether. The peptides were purified by prep HPLC (Thermo Spectra Physics) using a C18 reverse phase preparative column with a flow rate of $20 \mathrm{~mL} / \mathrm{min}$ in acetonitrile-water-TFA and water-TFA mixtures. Purity was analyzed by analytical HPLC (Dionex 1100) using a reverse phase C18 column at a flow rate of $1.5 \mathrm{~mL} / \mathrm{min}$ and LCMS (Thermo Surveyor 355). Samples of high purity ( $\geq 99 \%)$ were used in our studies.

For nanotube assembly, peptides were suspended in water to which acetonitrile was added to yield a $1 \mathrm{mM}$ solution with water-acetonitrile volumetric ratio of 10:90, 5:95 and 2:98 for $\mathrm{c}(\mathrm{KF})_{4}, \mathrm{c}(\mathrm{R} \underline{{ }})_{4}$ and $\mathrm{c}(\mathrm{H} \underline{\mathrm{F}})_{4}$, respectively. The samples were equilibrated in a closed Eppendorf for three weeks at room temperature. 


\section{S1.3 Atomic force microscopy (AFM)}

AFM samples were prepared by drop casting $2 \mu$ aliquots of a $1 \mathrm{mM}$ solution containing the peptide assemblies on silicon substrates with a $100 \mathrm{~nm}$ top oxide layer. Excess solution was pipetted out and the samples were dried overnight. Prior to peptide nanotubes deposition, the silicon wafers were cleaned using freshly prepared Piranha solution $\left(30 \% \mathrm{H}_{2} \mathrm{O}_{2}\right.$ in concentrated $\mathrm{H}_{2} \mathrm{SO}_{4}$ ) for 30 minutes (Caution: Piranha is a strong oxidant and reacts violently with many organic materials), followed by three washings in TDW, and drying under a nitrogen flow. AFM imaging was performed in tapping mode (Solver-Pro,NT-MDT, Russia) with non-contact tips (Multi75Al-G, BudgetSensors, Bulgaria, spring constant of $3 \mathrm{Nm}^{-1}$ and resonance frequency of $75 \mathrm{KHz}$ ). Background subtraction was performed by second order polynomial fitting using the NOVA AFM software. The NOVA software was also used to extract the peptide fiber's diameter as their height in cross section curves obtained from the topography images. Measurements of at least 30 cross sections of different fibrils from at least 4 different samples were averaged to obtain the width of each type of fiber with the error calculated as the standard deviation.

\section{S1.4 Structural studies}

Polarization modulation-infrared reflection-absorption spectroscopy (PM-IRRAS) was conducted on dried peptide film drop-casted from solution on gold substrates in a glove box (to minimize spreading on the surface), using a Thermo Scientific Nicoet 6700 FT-IR spectrometer with a liquid nitrogen cooled MCT-A detector with $8 \mathrm{~cm}^{-1}$ resolution. Each experiment was repeated at least 3 times for each peptide assembly. The spectra obtained for each peptide represents the average of 1000 scans. Spectra were processed for baseline correction and background noise removal using OMNIC 8.3 software (Thermo Scientific).

\section{S1.5 Electrical characterization of the peptide films}

For the electrical characterization of the peptide films, chips containing 6 sets of 500 $\mu \mathrm{m}$ wide and $50 \mathrm{~nm}$ thick palladium contacts with gaps of 3, 4, 5, 10 and $20 \mu \mathrm{m}$ were fabricated by electron beam sputtering and conventional photolithography on silicon wafers with a 100 $\mathrm{nm}$ thermal oxide top layer using a $10 \mathrm{~nm}$ titanium adhesive layer. Prior to peptide sample deposition the chips were cleaned by sonication in acetone, methanol and isopropanol for 15 minutes each, and dried under nitrogen flow. To eliminate contributions of surface hydroxyl groups to the conductivity, the chips were then silanized using triethoxy(octyl)silane (TEOS, Sigma Aldrich, deposition grade 98\%) in a dry nitrogen atmosphere for 45 minutes. The chips 
were sonicated in dehydrated absolute ethanol (HPLC grade, Bio-Lab) for 10 minutes to remove excess silane molecules, and then annealed in an oven at $110^{\circ} \mathrm{C}$ for 20 minutes. Contact angle measurements (Kruss $\mathrm{HmbH}$, Germany) indicated a contact angle $>95^{\circ}$ for the silanized chips. Samples were prepared by drop casting 2.5 or $5 \mu \mathrm{L}$ drops of freshly prepared peptide solutions on the devices in 4 or 2 repeating steps to yield final volume of $10 \mu \mathrm{L}$ dropcasted solution on the surface. Devices were then dried overnight at room atmosphere. Measurements were performed under controlled relative humidity of $60 \%$, monitored by a hygrothermometer (Extech), and maintained manually by mixing a dry and humidified flows of a gas mixture of $\mathrm{N}_{2}$ with $4.8 \% \mathrm{H}_{2}$. The hydrogen environment ensured hydrogenation of palladium making the contacts proton transparent. ${ }^{2-3}$

Electrical measurements were performed in a two points configuration in a probe station (Janis, USA). A 6430 Keithley source-meter was used for current- voltage (I-V) measurements. Measurements were performed with $0.05 \mathrm{mV}$ steps and $2 \mathrm{sec}$ sweep delay. The measurements were repeated on at least 3 chips for each peptide sample. Resistances were estimated from the inverse of the I-V slopes, averaged over at least 6 measurements with the standard deviation reported as the error. Solid-state impedance spectroscopy (IS) measurements were performed in the same probe station, using a Solartron 1260 frequency response analyzer and 1296 dielectric interface. Impedance spectra were recorded over a frequency range of $32 \mathrm{MHz}$ to $0.032 \mathrm{~Hz}$, with $\mathrm{DC}$ level of $0 \mathrm{~V}$ and $\mathrm{AC}$ amplitude of $100 \mathrm{mV}$. Equivalent electric circuit fitting was done with the EC-Lab (BioLogic) software using a nonlinear, least-square method.

\section{S1.6 Water uptake}

Water uptake was monitored as weight gain evaluated from frequency shifts in quartz crystal micro-balance measurements (Inficon Maxtek ${ }^{\circledR}$ RQCM, USA). For each sample, the weight of a dry peptide was measured after equilibration in nitrogen for 30 minutes. The sample was then equilibrated at $60 \%$ relative humidity and the change in the sample weight was recorded. The water/peptide molecular ratio was obtained using the molecular weight of water and the peptide. 


\section{S2 Additional experimental characterizations data}

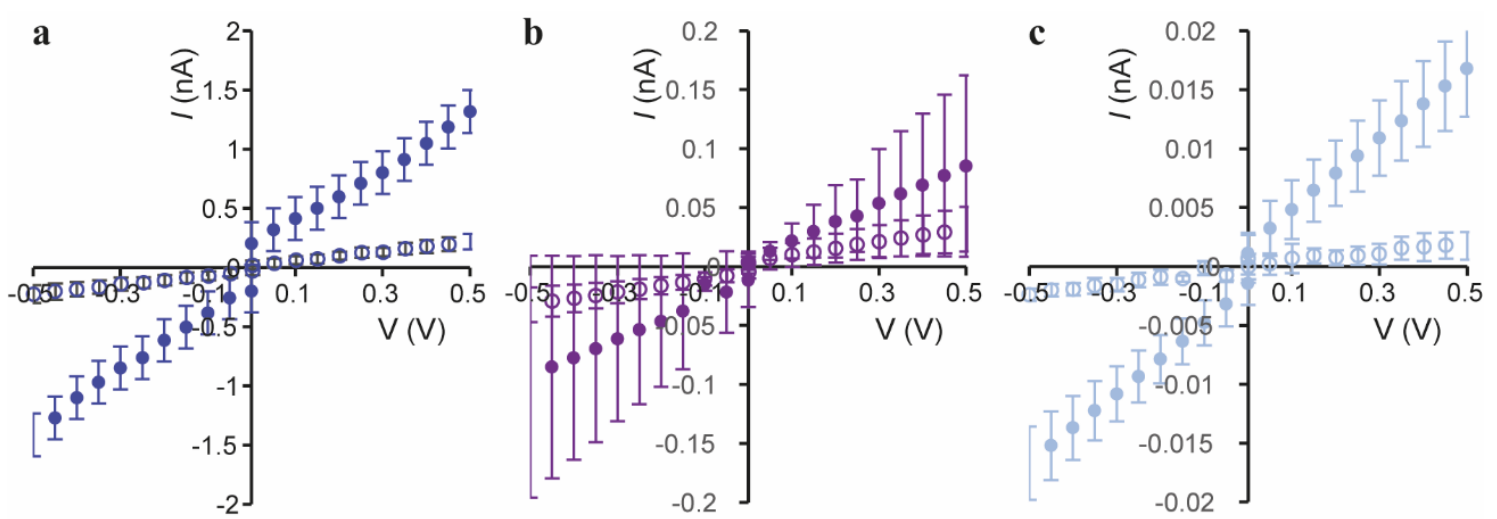

Figure S1. I-V curves of the three samples at $40 \%$ relative humidity of $\mathrm{H}_{2} \mathrm{O}$ (full circles) and $\mathrm{D}_{2} \mathrm{O}$ (empty circles) for a) $\left.c(K \underline{F})_{4}, b\right) c(R \underline{F})_{4}$ and c) $c(H \underline{F})_{4}$. Data is averaged over 4-6 measurements for each of the samples, and the error is provided as the standard deviation of the measurements.

Table S1. Equivalent circuit parameters extracted from fittings of IS data in Figure $2 \mathrm{~b}$. The equivalent circuit used for data fitting is depicted in the inset of Figure $2 \mathrm{~b}$. $R_{2}$ corresponds to the film resistance values depicted in Figure $2 d$.

\begin{tabular}{|l|l|l|l|}
\hline & $\mathrm{c}(\mathrm{KF})_{4}$ & $\mathrm{c}(\mathrm{RF})_{4}$ & $\mathrm{c}(\mathrm{HF})_{4}$ \\
\hline $\boldsymbol{R}_{\mathbf{1}}[\mathbf{\Omega}]$ & 47 & 40 & 58 \\
\hline $\boldsymbol{C}_{\mathbf{1}}[\mathrm{pF}]$ & 6 & 27 & 28 \\
\hline $\boldsymbol{Q}_{\mathbf{2}}\left[\mathrm{pF} * \mathbf{s e c}^{(\mathrm{a}-1)}\right]$ & 49 & 75 & 405 \\
$\boldsymbol{a}_{\mathbf{2}}$ & 1.0 & 1.0 & 0.9 \\
\hline $\boldsymbol{R}_{\mathbf{2}}[\mathrm{M} \Omega]$ & 27 & 550 & 2071 \\
\hline $\boldsymbol{Q}_{\mathbf{3}}\left[\mathrm{pF} * \sec ^{(\mathrm{a}-1)}\right]$ & 1457 & 260 & 283 \\
$\boldsymbol{a}_{\mathbf{3}}$ & 0.6 & 0.6 & 0.2 \\
\hline $\boldsymbol{R}_{\mathbf{3}}[\mathrm{G} \Omega]$ & 1 & 3 & 7 \\
\hline
\end{tabular}

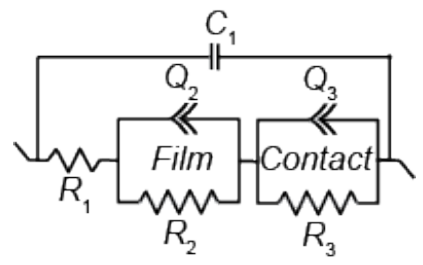


Table S2. Water uptake (WU) per peptide molecule for self-assembled peptide nanotube bundles, with respect to the sample in dry conditions (under nitrogen).

\begin{tabular}{|l|c|c|}
\hline Peptide & $\begin{array}{c}\text { Molecular } \\
\text { Weight (g/mole) }\end{array}$ & $\begin{array}{c}\text { WU }^{\mathbf{a}} \\
\mathbf{6 0 \%} \mathbf{R H}\end{array}$ \\
\hline c(KF)4 & 1101.41 & 3 \\
\hline c(RF)4 & 1213.47 & 5 \\
\hline c(HF)4 & 1137.28 & 8 \\
\hline
\end{tabular}

a Note this is an underestimation that does not take into account strongly bound water. 


\section{S3 Molecular Dynamics (MD) simulations}

\section{S3.1 Peptide systems}

Figure S2 shows the chemical structures of the cyclic peptides under study. These are the building blocks ("monomers") of the nanotubes that are studied theoretically and that form fibrils in the experiments.
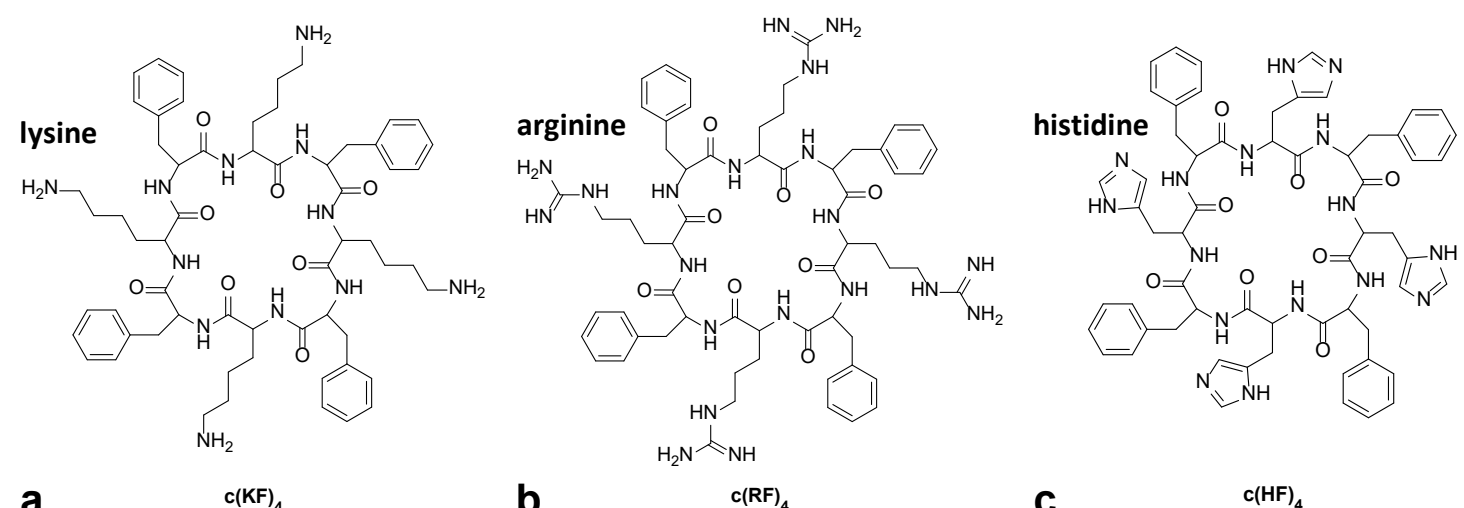

b

$\mathrm{c}(\mathrm{RF})_{4}$

C

$\mathrm{c}(\mathrm{HF})_{4}$
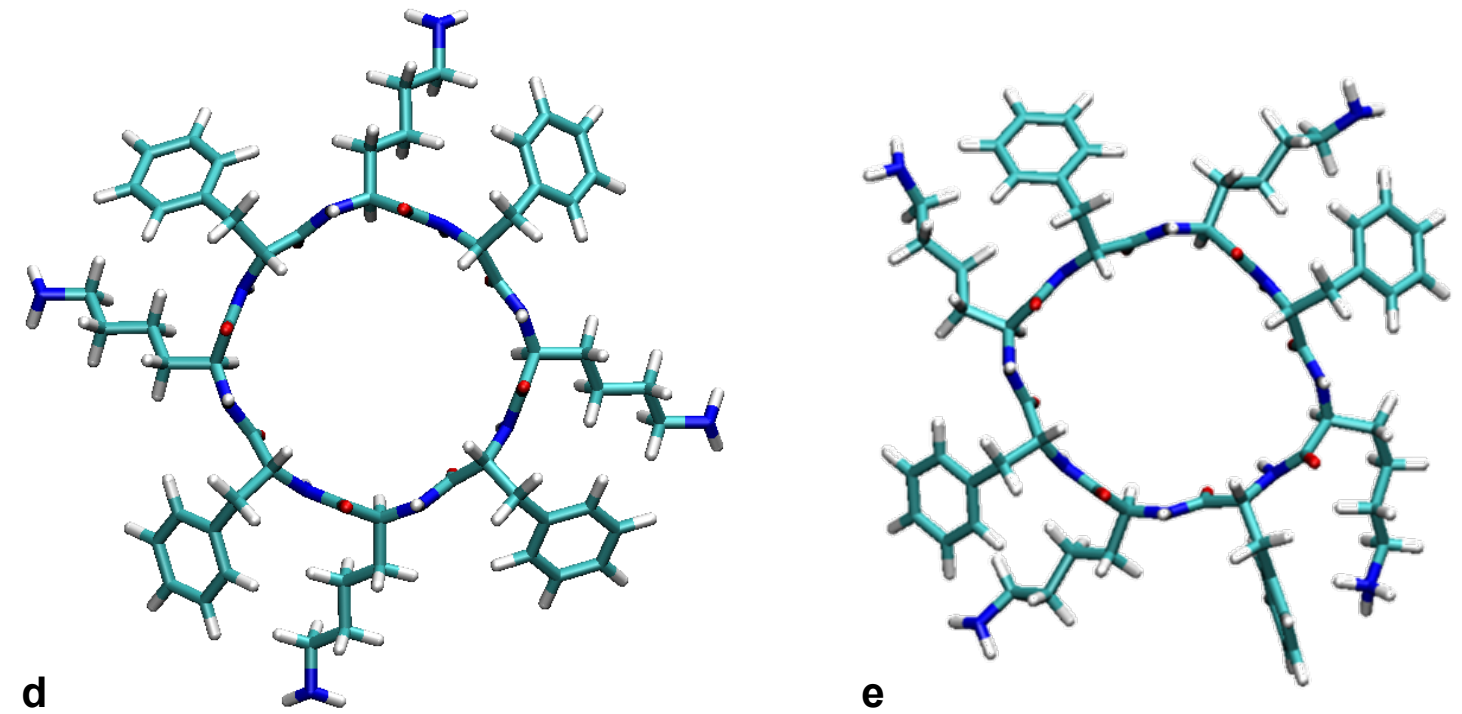

Figure S2. Structures of the cyclic peptides investigated: $(a) c(K \underline{F})_{4},(b) c(R \underline{F})_{4}$, and $(c) c(H \underline{F})_{4}$. These oligopeptides contain four phenylalanine amino acids at D-positions that are alternated with (a) lysine, (b) arginine and (c) histidine at L-positions. The structures were optimized using DFT with the B3LYP/6-31g* computational setup. The optimized structures of $(\mathrm{d}) \mathrm{c}(\mathrm{K} \underline{\mathrm{F}})_{4}$ and $(\mathrm{e}) \mathrm{c}(\mathrm{K} \underline{\mathrm{F}})_{4} \mathrm{H}^{+}$monomers are shown.

The monomer geometries were optimized using density functional theory (DFT) with the B3LYP hybrid exchange-correlation functional ${ }^{4-7}$ and the $6-31 \mathrm{~g}^{*}$ Pople $^{8}$ basis set. The monomers were stacked in an antiparallel structure at an initial average distance of $4.6 \AA$ to 
form hexamers (Figure 3) or decamers (vide infra). We added an excess proton to one of the two central (i.e., third and fourth) peptide rings to study the proton transfer (PT) between amino acid side chains. The use of the internal monomers for the PT analysis aimed to limit the structural fluctuations of the molecular framework around the proton donor $(D)$ and acceptor $(A)$, especially in the MD simulations of the solvated systems. In fact, we compared our theoretical analysis with the results of experiments in which the peptide rings have several structural constraints, including those deriving from the micrometric length and the bundling of the peptide nanotubes into fibrils. To partly model these constraints, we increased the rigidity of the systems in our MD simulations by constraining the terminal rings in the nanotubes. No enhancement in rigidity of the central monomers resulted from using cyclic peptide decamers instead of hexamers, as is detailed in Section S2.3. We performed DFT computations using the NWChem software package. ${ }^{9}$

\section{S3.2 MD simulations}

MD simulations were performed using the NAMD code, version $2.11 \mathrm{~b} 1 .^{10}$ The results reported in the article were obtained from MD simulations at constant volume and at 298.15 $\mathrm{K}$ (thus sampling a canonical or NVT ensemble), using the CHARMM36 force field, ${ }^{11}$ which was shown to be particularly appropriate for protein simulations. ${ }^{12}$ The peptide systems with one excess proton were neutralized with $\mathrm{a} \mathrm{Cl}^{-}$ion. We used the multilevel summation method $(M S M)^{13}$ for full electrostatics calculations. As the simulations in vacuum that produced the results presented in the article did not make use of periodic boundary conditions, the use of an NVT ensemble with MSM full electrostatics (which avoids the use of the fast Fourier transform) allowed us to simulate the system consistently in the vacuum and fully solvated conditions. The force constant for the restraining harmonic potential was set to 10 $\mathrm{kcal} /\left(\mathrm{mol} \cdot \AA^{2}\right)$ and the pertinent exponent was set to 2 . All simulations were carried out using Langevin dynamics (also for $\mathrm{H}$ atoms) with a damping coefficient of $1 \mathrm{ps}^{-1}$.

In the MD simulations of the solvated systems, TIP3P water ${ }^{14}$ was restrained to the simulated region using spherical boundary conditions, with solvation radii of 30 and $40 \AA$ for the peptide hexamers and decamers, respectively (Figure S3 shows a snapshot of a solvated peptide nanotube during its MD simulation). We constrained the $\mathrm{H}-\mathrm{H}$ and $\mathrm{O}-\mathrm{H}$ distances in the water molecules using the SHAKE algorithm. We used a scaling factor of 1 for the 1-4 electrostatic interactions. The van der Waals interactions were cut off at a distance of $12 \AA$, 
and the switching function was used in the range 10-12 $\AA$. The periodic computation of the interaction energy was performed with a maximum non-bonded interaction distance of $14 \AA$. The reassignment of the atoms for the computation of the non-bonded interactions was performed every $10 \mathrm{MD}$ steps, while the full electrostatic interaction energy was evaluated each 2 timesteps. A timestep of $1 \mathrm{fs}$ was used.

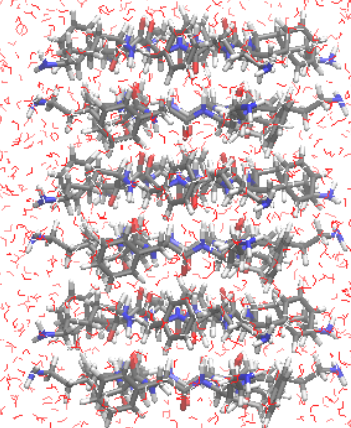

Figure S3. MD snapshot of a cyclic-peptide hexamer in water solvent, which shows the effect of using the spherical boundary conditions on the distribution of water around the nanotube.

After $10^{4}$ geometry optimization steps of the solvent only (for the systems in water), the coordinates of the unconstrained cyclic-peptide hexamers were released for further $10^{4}$ steps of energy minimization. Afterwards, we carried out $5 \mathrm{~ns}$ of preliminary equilibration at constant temperature (using the Langevin dynamics), followed by $45 \mathrm{~ns}$ of molecular dynamics. The last $40 \mathrm{~ns}$ of this MD were used for the PT analysis. Similar MD simulations were performed for the systems with constrained edge peptides. In such simulations, the average distance between the backbone atoms of the external peptides was set to the value that prevalently emerged from the simulations of the free hexamers. More precisely, the average of the axial distance between the external peptides over the MD snapshots (either in vacuum or in water) was $4.8 \AA$ for the $\mathrm{c}(\mathrm{RF})_{4}$ nanotube and $4.9 \AA$ for the two other systems (that is, as expected, slightly larger than the $4.73 \AA$ spacing observed in hundreds of nanometers-long peptide filaments ${ }^{15}$ ). The MD production runs were extended to 200 ns for the decamers. 


\section{S3.3 MD analysis}

Figure S4 shows examples of RMSD evolution along the MD simulations of the nanotubes (note the large values of the RMSD for the more flexible peptide decamer in water).
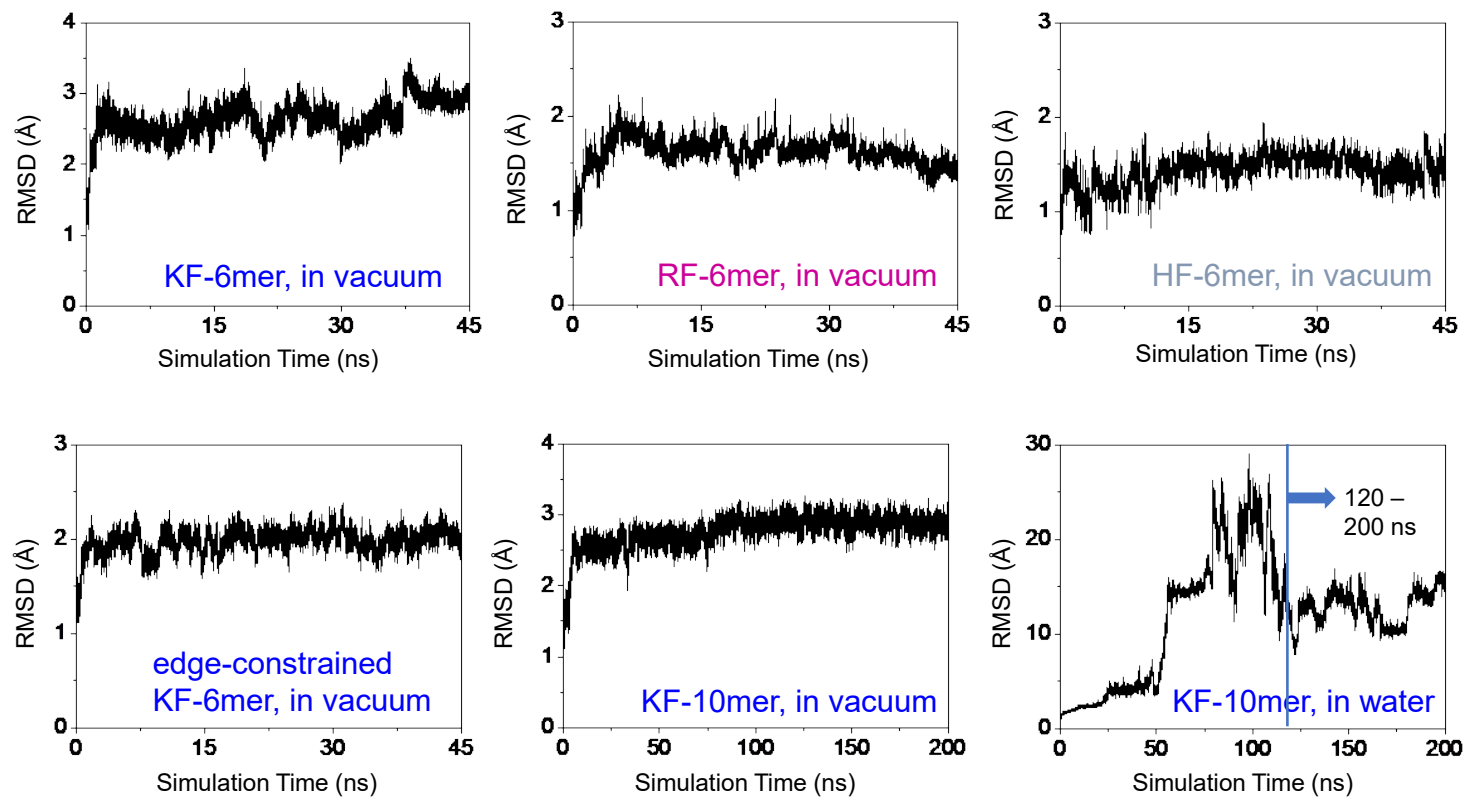

Figure S4. Examples of RMSD evolutions for some of the peptide systems studied.

Figures S5, S6, and S7 show the structures of the peptide nanotubes in the first, middle and last snapshots from their respective MD production runs. Figures $S 7 b, d, f$ show that the simulations of the solvated $\mathrm{c}(\mathrm{H} \underline{\mathrm{F}})_{4}$ stacks lead to breaking of the central portions of the structures, even when the two edge rings are constrained. For this reason, we limited the analysis of PT in water, and hence its comparison to PT in vacuum, to the $c(K \underline{F})_{4}$ and $c(R \underline{F})_{4}$ systems, for which the internal rings (in particular, the peptide rings involved in the PT analysis) remained stacked throughout the MD simulations. Figures S5f, S6f show that, in water, the external portions of the cyclic-peptide decamers are not structurally more stable than those of the corresponding hexamers, owing to their higher flexibility compared to the shorter stacks, and the fact that the longer peptide stacks are not bundled as in the experiments. In particular, the peptide rings beyond a certain stack length can detach from the stable nanotube core and fluctuate in the solvent. This is a dramatic consequence of the water effect on the nanotubes described in the article. The structures of the constrained $c(K \underline{F})_{4}$ and $c(\mathrm{RF})_{4}$ stacks are, instead, very stable in both dry and full-solvation conditions. 

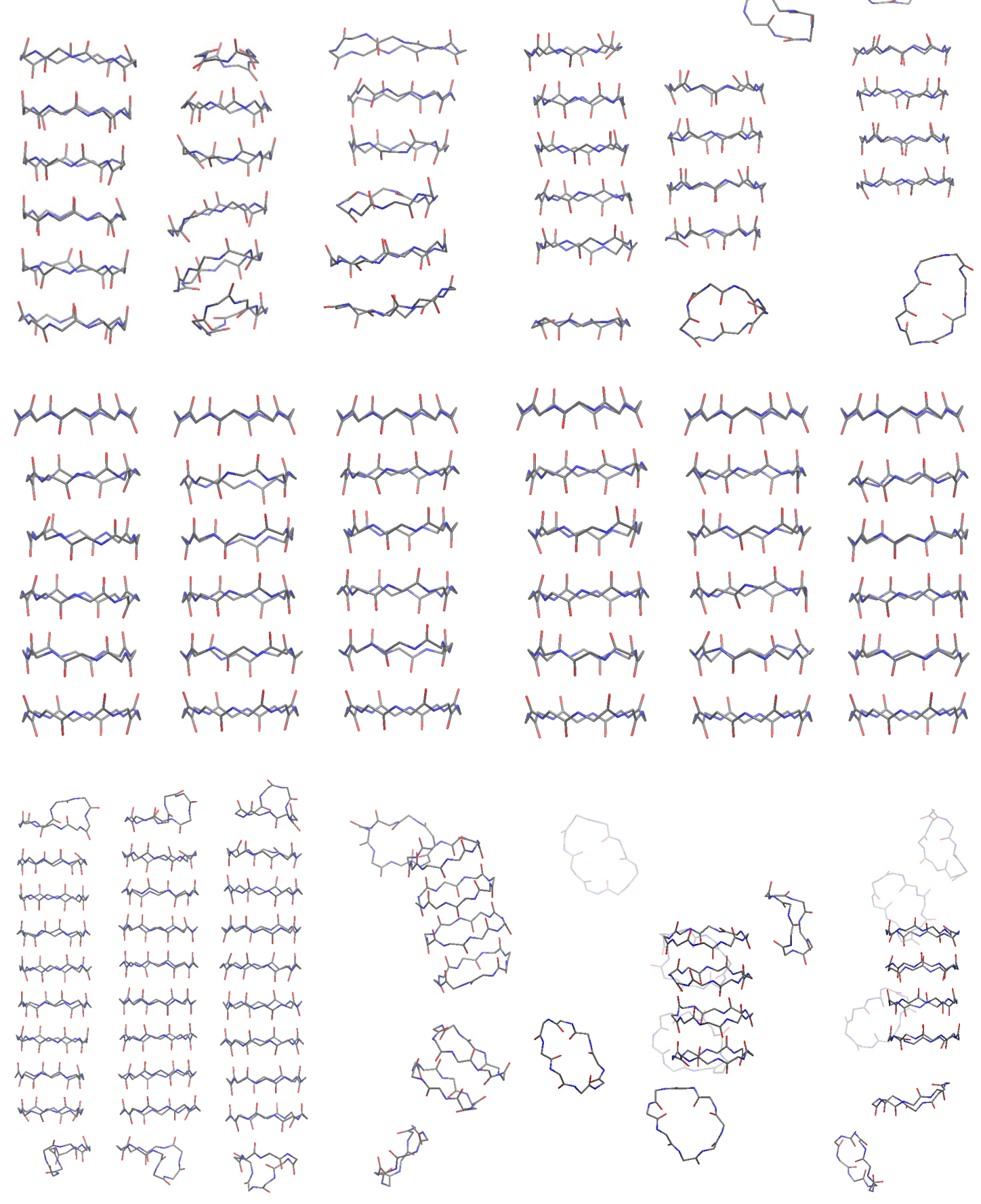

Figure S5. MD snapshots of the free $(a, b)$ and edge-constrained $(c-d) c(K F)_{4}$ hexamers, in vacuum $(a, c)$ and in water (b, d), and of the dry (e) and solvated (f) decamer (without structural constraints). The snapshots (from left to right) are taken at 5, 25, and $45 \mathrm{~ns}$ of the hexamer MD simulation, and at 120,160, and $200 \mathrm{~ns}$ of the decamer MD. Only the backbone heavy atoms of the cyclic peptides are shown. 
Whate spanat perdy

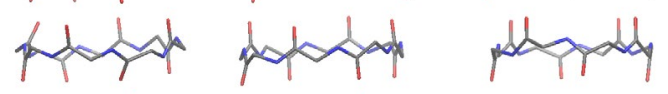

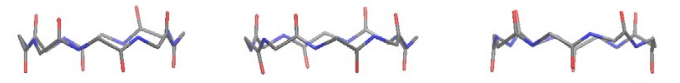

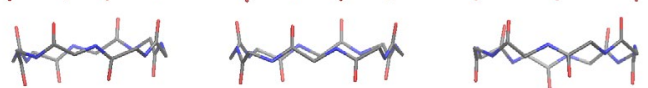

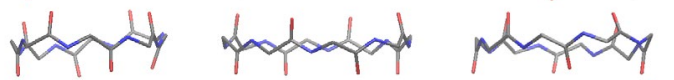

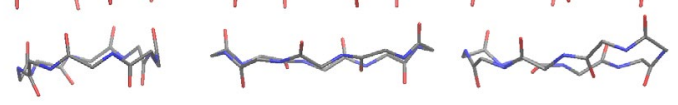

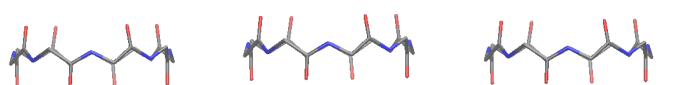

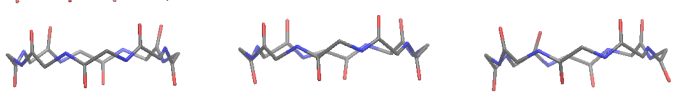

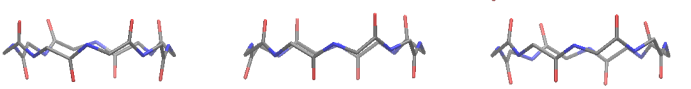

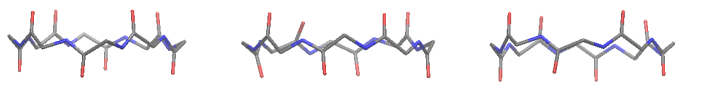

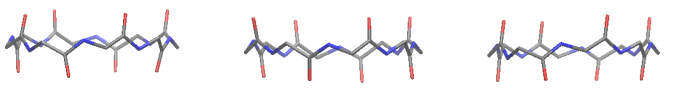

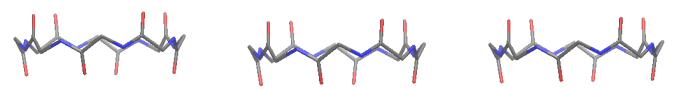

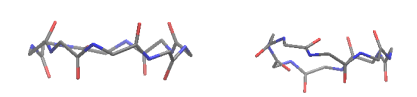
reate spded parep maxpd propty<smiles></smiles>

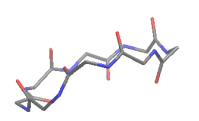
hrobst

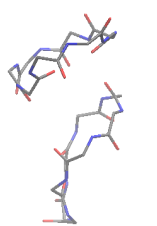

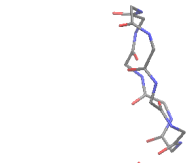

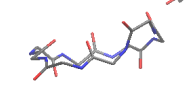
and mposts<smiles>c1ccc(C23CC4CC(C2)C(C4)C3)cc1</smiles>

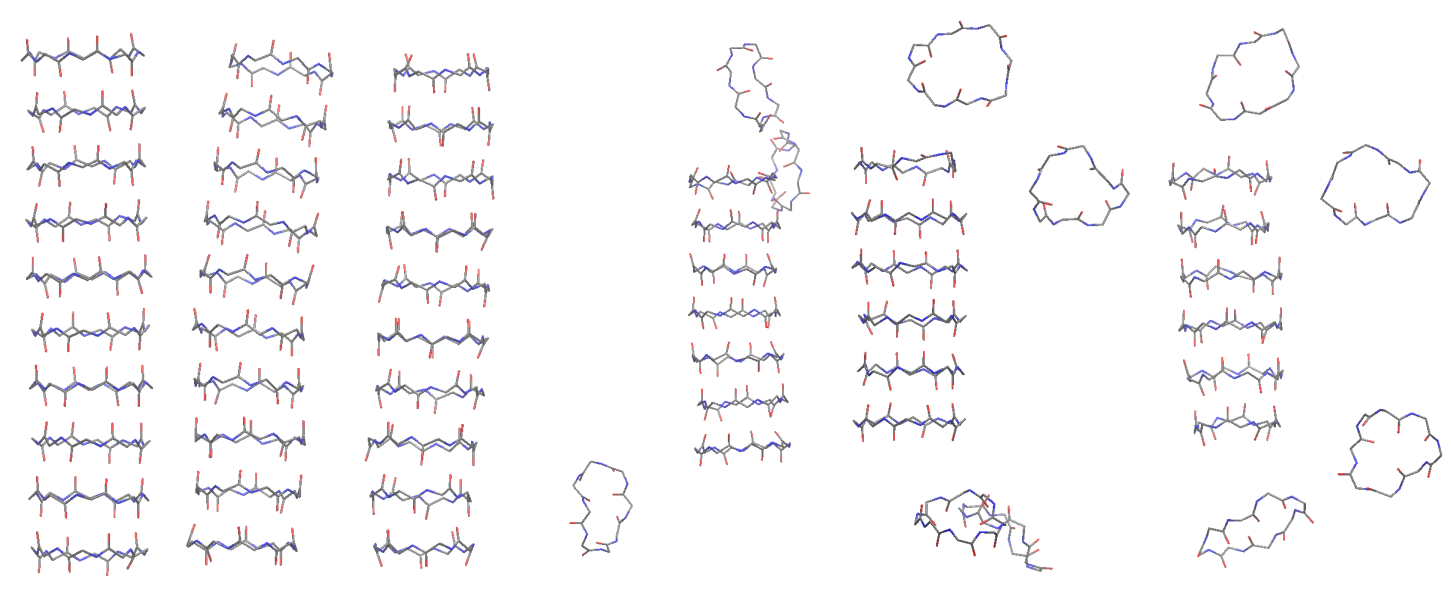

Figure S6. MD structural snapshots of the $c(\mathrm{RF})_{4}$ systems represented in the same order as in Figure S6. In this case, the first MD snapshot of the decamer is taken at $100 \mathrm{~ns}$ of the production run, and the other two MD snapshots correspond to 150 and 200 ns. 

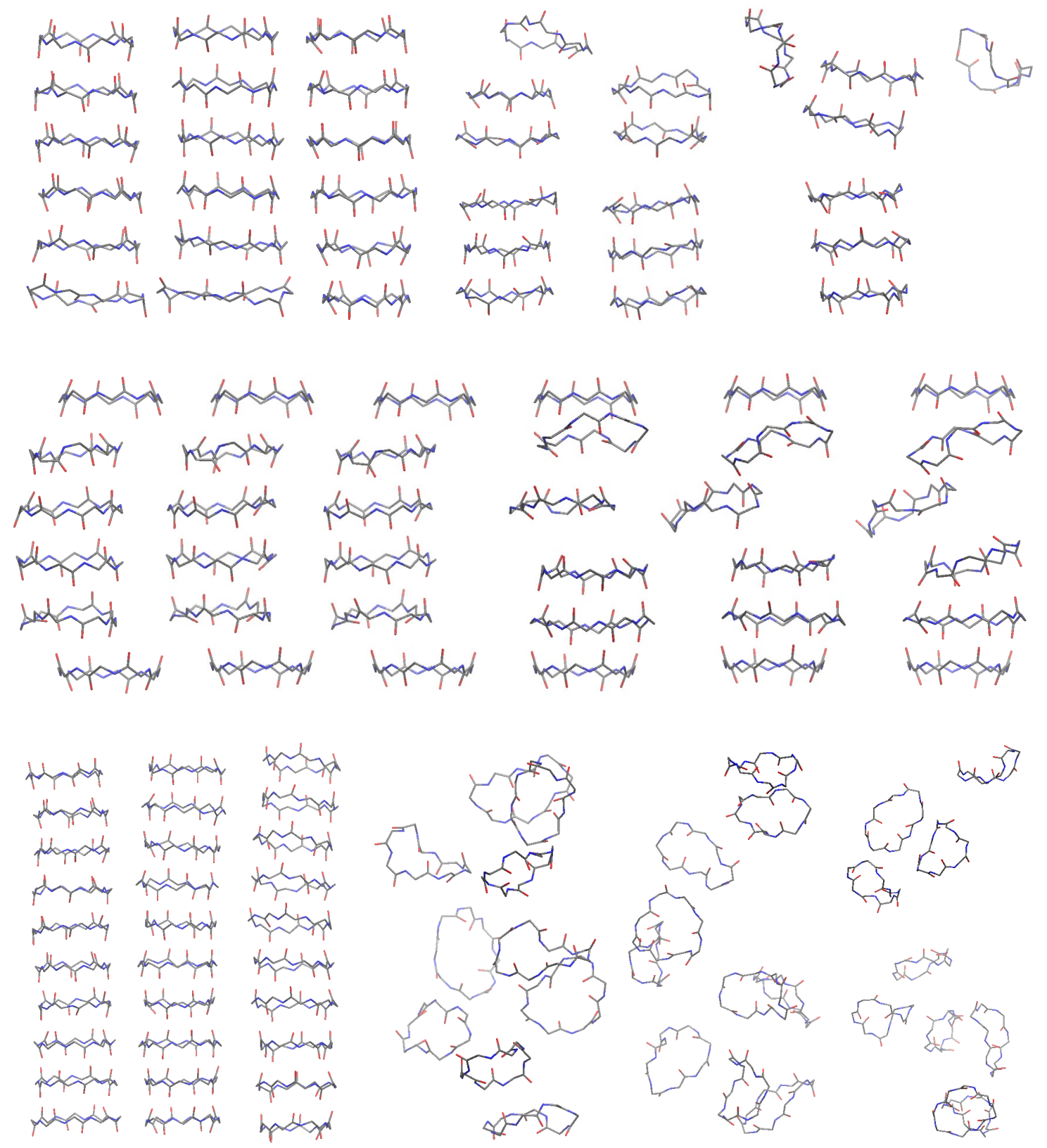

Figure S7. MD structural snapshots of the $\mathrm{c}(\mathrm{HE})_{4}$ systems represented in the same order as in Figure S6. Here, the first snapshot of the decamer is taken at $80 \mathrm{~ns}$ of the MD run, and the other two MD snapshots correspond to 140 and 200 ns.

\section{S4 Theoretical modeling}

\section{S4.1 Proton couplings and transfer rates}

We assumed that the proton coupling is proportional to the proton wave function overlap. Eq 7.11 of ref ${ }^{16}$ was used to estimate this overlap in snapshots separated by 1 ps along the MD simulation. We assumed nonadiabatic PT because of the short-range nature of PT. These assumptions, combined with the other approximations described in the article, 
$K$ proportional to the pertinent proton wave function overlaps. The effective PT rates to be used in the kinetic model (see next section) were obtained by weighing the mean-square overlaps with the fractions of snapshots in which the hydrogen bonding allowed the occurrence of PT. That is, for the $1 d$ or $2 d$ PT process in the MD simulation of a given system, it is

$$
\begin{aligned}
& k=k_{0} \frac{1}{N_{\text {tot }}} \sum_{i=1}^{N_{\mathrm{H}-\text { bond }}} S_{\mathrm{D}-\mathrm{A}}^{2}=k_{0} \frac{N_{\mathrm{H}-\text { bond }}}{N_{\text {tot }}}\left(\frac{1}{N_{\mathrm{H}-\text { bond }}} \sum_{i=1}^{N_{\mathrm{H}-\text { bond }}} S_{\mathrm{D}-\mathrm{A}}^{2}\right) \\
& =f_{\mathrm{H}-\text { bond }} k_{0}\left\langle S_{\mathrm{D}-\mathrm{A}}^{2}\right\rangle_{\mathrm{H}-\text { bond }} \equiv f_{\mathrm{H}-\text { bond }} K
\end{aligned}
$$

where $k$ denotes the effective PT rate, $N_{\text {tot }}$ is the total number of snapshots selected for PT analysis, $N_{\text {H-bond }}$ is the number of snapshots with D-A hydrogen bonding, $f_{\text {H-bond }}=N_{\text {H-bond }} / N_{\text {tot }}$. In the model, $k_{0}$ is a (not evaluated) proportionality constant. In the presence of a water (W) bridge, the effective rate for a two-step PT process is (as in a two-step electron transfer ${ }^{17}$ )

$$
\begin{aligned}
& k=\left(\frac{1}{k_{\mathrm{D}-\mathrm{W}}}+\frac{1}{k_{\mathrm{W}-\mathrm{D}}}\right)^{-1}=f_{\mathrm{H}-\mathrm{bond}} k_{0}\left\langle\left(\frac{1}{S_{\mathrm{D}-\mathrm{W}}^{2}}+\frac{1}{S_{\mathrm{W}-\mathrm{A}}^{2}}\right)^{-1}\right\rangle_{\mathrm{H}-\text { bond configurations }} \\
& =f_{\mathrm{H}-\mathrm{bond}} k_{0}\left\langle\Sigma_{\mathrm{D}-\mathrm{A}}^{2}\right\rangle_{\mathrm{H}-\text { bond configurations }}
\end{aligned}
$$

where we used the same $k_{0}$ value for the two PT steps, based on the approximations described in the article, which include disregarding the backward (with respect to the direction fixed by the applied voltage) PT rates.

\section{S4.2 Proton-transport model}

The proton transport model described in this section is illustrated in Figure $3 b$, which is reproduced below for convenience. $J$ is the proton flux (that is, the proton current per peptide) through a peptide nanotube that is sustained by the source (or "left", L in Figure S8) and drain (or "right", R) electrodes, in the steady-state approximation. From a macroscopic point of view, such an approximation is local in time, as the current is not a constant in the experiments, but it can be assumed as a good approximation on the nanoscale over sufficiently short time scales. 


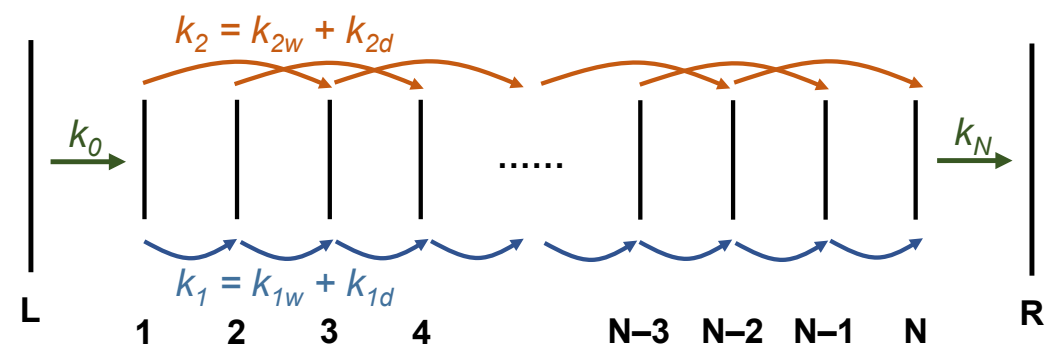

Figure S8. Schematic of the proton transport model leading to eq 2.

We assume that all cyclic peptides are equivalent (that is, we are neglecting the influence of the electrodes on the nearby peptides) and hence are characterized by the same effective rates $k_{1}$ and $k_{2}$. We expect this to be a reasonable approximation for any nanoscale portion of a much longer peptide nanotube within a sample. Furthermore, this may also be a good approximation for peptide rings near the (composite) electrodes, since the inter-peptide PT should depend largely on the local environment between the D and A amino acid side chains. We consider a single excess proton in the cyclic peptide chain (the model still applies, apart from a proportionality constant in the expression for $J$, if more protons are transported independently across the system; see the discussion in the article), and we neglect back PT rates (this approximation is based on the presence of a potential bias and simplifies the expression for the proton flux).

We call $P_{n}$ the probability that site $n$ (that is, peptide ring $n$ ) is occupied by the excess proton. The effective rates $k_{0}$ and $k_{N}$ ( $N$ is the number of peptide cycles stacked in the proton transport chain) are conveniently introduced in the kinetic model to relate the overall incoming and outgoing proton fluxes to the proton occupation probability of the system. The master equation of the system is

$$
\left\{\begin{array}{l}
\frac{d P_{1}}{d t}=J-P_{1}\left(k_{1}+k_{2}\right)=0 \\
\frac{d P_{2}}{d t}=P_{1} k_{1}-P_{2}\left(k_{1}+k_{2}\right)=0 \\
\frac{d P_{n}}{d t}=P_{n-2} k_{2}+P_{n-1} k_{1}-P_{n}\left(k_{1}+k_{2}\right)=0 \quad(3 \leq n \leq N-2) \\
\frac{d P_{N-1}}{d t}=P_{N-3} k_{2}+P_{N-2} k_{1}-P_{N-1} k_{1}=0 \\
\frac{d P_{N}}{d t}=P_{N-2} k_{2}+P_{N-1} k_{1}-J=0
\end{array}\right.
$$


where

$$
J=\left(k_{1}+k_{2}\right) P_{1}=k_{N} P_{N}
$$

Eqs S3 and S4 give

$$
\left\{\begin{array}{l}
P_{1}=\frac{J}{k_{1}+k_{2}} \\
P_{2}=\frac{k_{1}}{k_{1}+k_{2}} P_{1} \\
P_{n}=\frac{k_{2} P_{n-2}+k_{1} P_{n-1}}{k_{1}+k_{2}} \quad(3 \leq n \leq N-2) \\
P_{N-1}=P_{N-2}+\frac{k_{2}}{k_{1}} P_{N-3} \\
P_{N}=\frac{k_{2} P_{N-2}+k_{1} P_{N-1}}{k_{N}}
\end{array}\right.
$$

Eq S5 yields the recursive relation

$$
\begin{aligned}
& P_{n}-P_{n-1}=\frac{k_{2} P_{n-2}+k_{1} P_{n-1}}{k_{1}+k_{2}}-P_{n-1}=-f\left(P_{n-1}-P_{n-2}\right) \\
& =(-f)^{n-2}\left(P_{2}-P_{1}\right)=(-f)^{n-2}\left(\frac{k_{1}}{k_{1}+k_{2}}-1\right) P_{1}=(-f)^{n-1} P_{1} \quad(3 \leq n \leq N-2)
\end{aligned}
$$

with

$$
f=\frac{k_{2}}{k_{1}+k_{2}}
$$

The use of the expression for $P_{2}$ in eq S5 shows that eq S6a also applies to $n=2$. Then, adding the quantities $P_{i+1}-P_{i}$ for $i=1, \ldots, n$, we find

$$
P_{n}-P_{1}=P_{1} \sum_{i=1}^{n-1}(-f)^{i} \Rightarrow P_{n}=\sum_{i=0}^{n-1}(-f)^{i} \quad(1 \leq n \leq N-2)
$$

Substituting eq S7 for $n=N-2$ and $n=N-3$ into the penultimate relation of eq S5, we find

$$
P_{N-1}=P_{1}\left[\sum_{i=0}^{N-3}(-f)^{i}+\frac{f}{1-f} \sum_{i=0}^{N-4}(-f)^{i}\right]
$$

Using eq S4, or combining eq S8 with eq S7 for $n=N-2$, we have

$$
P_{N}=P_{1} \frac{k_{1}+k_{2}}{k_{N}}
$$


Next, we consider the peptide bridge as a whole. In the kinetic model, the transport of the excess proton is unidirectional, and the left electrode continuously supplies a new proton as each previous one is delivered to the right electrode. Thus, the probability that a proton is either on the contact electrode surface or on the peptide bridge is always 1 . Therefore, we find the charge conservation relation

$$
P_{B} k_{N}=\left(1-P_{B}\right) k_{0} \Rightarrow P_{B}=\sum_{n=1}^{N} P_{i}=\frac{k_{0}}{k_{0}+k_{N}}
$$

where $P_{B}$ is the total occupation probability of the peptide bridge. Substituting the expressions of the site occupation probabilities into eq S10, we get

$$
P_{1}=\frac{k_{0}}{k_{0}+k_{N}}\left[\sum_{j=0}^{N-3} \sum_{i=0}^{j}(-f)^{i}+\sum_{i=0}^{N-3}(-f)^{i}+\frac{f}{1-f} \sum_{i=0}^{N-4}(-f)^{i}+\frac{k_{1}+k_{2}}{k_{N}}\right]^{-1}
$$

Since $\sum_{i=0}^{n} x^{n}=\frac{1-x^{n+1}}{1-x}$ and $|x|<1$, eq S11 becomes,

$$
\begin{aligned}
& P_{1}=\frac{k_{0}}{k_{0}+k_{N}} \frac{1+f}{N-1}\left[1+\frac{2 f}{1-f^{2}} \frac{1-(-f)^{N-1}}{N-1}+\frac{k_{1}+k_{2}}{k_{N}} \frac{1+f}{N-1}\right]^{-1} \\
& \cong \frac{k_{0}}{k_{0}+k_{N}} \frac{1+f}{N-1}=r \frac{k_{1}+2 k_{2}}{k_{1}+k_{2}}
\end{aligned}
$$

in which the dimensionless factor $r$ depends on the electrode-peptide interfaces and on the length of the polypeptide. The approximate expression in eq S12 is obtained for sufficiently large $N$, since $f<1$. Finally,

$$
J=P_{1}\left(k_{1}+k_{2}\right) \cong r\left(k_{1}+2 k_{2}\right)
$$

which yields eq 2 using the rate expressions in the previous section.

\section{S5 Kinetic analysis of the MD snapshots}

Table S3 shows the values of the normalized proton fluxes $J / J^{0}$ through $\mathrm{c}(\mathrm{RF})_{4}$ and $\mathrm{c}(\mathrm{HE})_{4}$ stacks with respect to the $\mathrm{c}(\mathrm{KE})_{4}$ stack. We also report the $J / J^{0}$ value for $\mathrm{c}(\mathrm{R} \underline{\mathrm{F}})_{4}$ in water obtained from comparative MD simulations at constant temperature and pressure using periodic boundary conditions, with a water layer of $12 \AA$ on each side of the peptide stack in the unit cell. This value differs from the one resulting from the MD simulations in spherical 
boundary conditions, but confirms the large gap in $J / J^{0}$ through the solvated $c(K \underline{K})_{4}$ and $c(R \underline{F})_{4}$ systems. The parameters inserted into eq 2 to determine the proton fluxes are reported in Table S4.

Table S3. $J / J^{0}$ values shown in Figures $4 a, 4 d$, and $5 a$. The $J / J^{0}$ value for $c(R \underline{R F})_{4}$, with respect to $c(K \underline{K})_{4}$, using periodic boundary conditions is also reported.

\begin{tabular}{l|lc}
\hline Normalized proton flux $J / J^{\mathbf{0}}$ with respect to $\mathbf{c}(\mathbf{K F})_{\mathbf{4}}$ & $\mathbf{c}(\mathbf{R F})_{4}$ & $\mathbf{c}(\mathbf{H F})_{4}$ \\
\hline free hexamer in vacuum & 0.29 & 0.93 \\
free hexamer in water & $7.3 \cdot 10^{-4}$ & - \\
Free hexamer in water, periodic boundary conditions & $4.6 \cdot 10^{-3}$ & - \\
edge-constrained hexamer in vacuum & 0.24 & 0.38 \\
\hline
\end{tabular}

Table S4. Parameters used in eq 2 to determine the proton fluxes for the free hexamer in vacuum and in solvent, and for the edge-constrained hexamer in vacuum. The values of the mean-square overlaps, which are proportional to the respective proton couplings, are normalized to the highest value among the different types of PT processes for the given kind of system in the leftmost column. For example, $\left\langle S_{\mathrm{D}-\mathrm{A}}^{2}\right\rangle_{1 d}$ is $1.88 \cdot 10^{-11}, 4.61 \cdot 10^{-12}$ and $9.80 \cdot 10^{-12}$ for the free $\mathrm{c}(\mathrm{KF})_{4}, \mathrm{c}(\mathrm{RF})_{4}$ and $\mathrm{c}(\mathrm{HE})_{4}$ hexamers in vacuum, respectively, and hence the values 1 , 0.25 , and 0.52 are reported in the order.

\begin{tabular}{lr|lll}
\hline & & $\mathbf{C}(\mathbf{K} \underline{\mathbf{F}})_{4}$ & $\mathbf{c}(\mathbf{R F})_{4}$ & $\mathbf{c}(\mathbf{H F})_{4}$ \\
\hline free hexamer in vacuum & $f_{1 d}$ & 0.44 & 0.52 & 0.79 \\
& $\left\langle S_{\mathrm{D}-\mathrm{A}}^{2}\right\rangle_{1 d}$ & 1 & 0.25 & 0.52 \\
free hexamer in water & $f_{1 d}$ & $5.5 \cdot 10^{-3}$ & $1.0 \cdot 10^{-3}$ & - \\
& $\left\langle S_{\mathrm{D}-\mathrm{A}}^{2}\right\rangle_{1 d}$ & 0.79 & 0.09 & - \\
& $f_{2 d}$ & 0.089 & 0 & - \\
& $\left\langle S_{\mathrm{D}-\mathrm{A}}^{2}\right\rangle_{2 d}$ & 1 & - & - \\
& $f_{1 w}$ & 0.014 & $2.5 \cdot 10^{-4}$ & - \\
& $\left\langle\Sigma_{\mathrm{D}-\mathrm{A}}^{2}\right\rangle_{1 w}$ & 0.04 & 0.18 & - \\
& $f_{2 w}$ & $6.3 \cdot 10^{-3}$ & 0 & - \\
& $\left\langle\Sigma_{\mathrm{D}-\mathrm{A}}^{2}\right\rangle_{2 w}$ & 0.07 & - & - \\
$f_{1 d}$ & 0.53 & 0.48 & 0.71 \\
\hline & $\left\langle S_{\mathrm{D}-\mathrm{A}}^{2}\right\rangle_{1 d}$ & 1 & 0.27 & 0.29 \\
\hline
\end{tabular}


The values of the normalized proton currents, and hence the related conclusions, are not affected by the duration of the MD simulations. Table S5 compares the $J / J^{0}$ values for the free hexamers in vacuum and in water shown in Tables S3 with the values $\left(J / J^{0}\right)_{30}$ and $\left(J / J^{0}\right)_{10}$ obtained using the last 30 ns and 10 ns of MD simulation, respectively.

Table S5. Proton currents $J$ through the $c(R \underline{R})_{4}$ and $c(H \underline{F})_{4}$ nanotubes, normalized to the value, $J_{0}$, in the $c(K \underline{K})_{4}$ nanotube, for the free hexamers in vacuum and in water. $J / J^{0},\left(J / J^{0}\right)_{30}$, and $\left(J / J^{0}\right)_{10}$ denote the relative proton fluxes obtained using the last 40,30 , and 10 ns of MD simulation, respectively.

\begin{tabular}{l|lllllc}
\hline & $\mathbf{C ( R F ) _ { 4 }}$ & \multicolumn{5}{c}{$\mathbf{c ( H F})_{4}$} \\
& $J / J^{0}$ & $\left(J / J^{0}\right)_{30}$ & $\left(J / J^{0}\right)_{10}$ & $J / J^{0}$ & $\left(J / J^{0}\right)_{30}$ & $\left(J / J^{0}\right)_{10}$ \\
\hline free hexamer in vacuum & 0.29 & 0.13 & 0.27 & 0.93 & 0.40 & 0.94 \\
free hexamer in water & $7.3 \cdot 10^{-4}$ & $6.3 \cdot 10^{-4}$ & $6.7 \cdot 10^{-4}$ & - & - & - \\
\hline
\end{tabular}

The $c(\mathrm{~K} \underline{\mathrm{F}})_{4}$ stack is more conductive than the other two systems also regarding electron transfer, as shown in Figure S9.

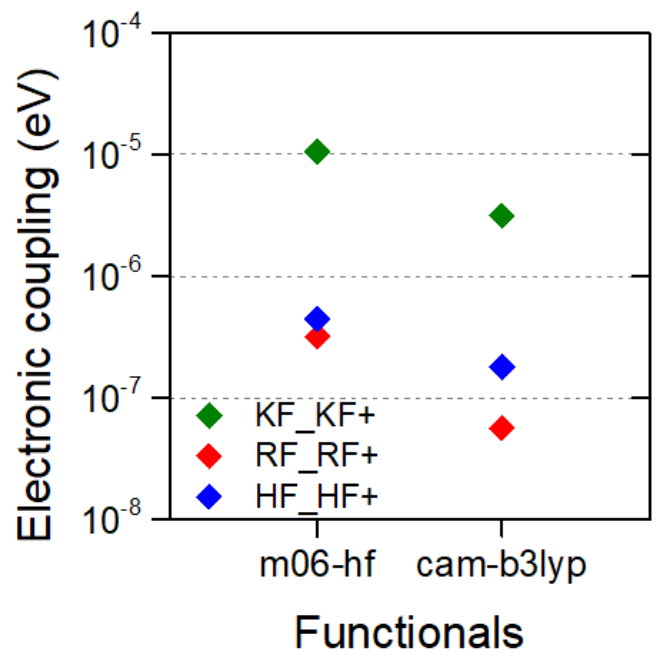

Figure S9. Electronic coupling for electron-hole transfer in the $\mathrm{c}(\mathrm{LF})_{4}-\mathrm{c}(\mathrm{LF})_{4}^{+}$redox couples ( $\left.\mathrm{L}=\mathrm{K}, \mathrm{R}, \mathrm{H}\right)$ resulting from DFT implementations of the method in refs ${ }^{18}{ }_{-}^{19}$, with the M06-HF ${ }^{20-21}$ and CAM-B3LYP ${ }^{22}$ exchangecorrelation functionals and the 6-31g* basis set. 


\section{S6 References}

1. Chan, W.; White, P., Fmoc Solid Phase Peptide Synthesis: A Practical Approach. Oxford University Press: New York, 2000.

2. Amit, M.; Roy, S.; Deng, Y. X.; Josberger, E.; Rolandi, M.; Ashkenasy, N., Measuring Proton Currents of Bioinspired Materials with Metallic Contacts. ACS Appl. Mater. Inter. 2018, 10, 1933-1938.

3. Zhong, C.; Deng, Y. X.; Roudsari, A. F.; Kapetanovic, A.; Anantram, M. P.; Rolandi, M., A Polysaccharide Bioprotonic Field-Effect Transistor. Nat. Commun. 2011, 2, 476.

4. Vosko, S. H.; Wilk, L.; Nusair, M., Accurate Spin-Dependent Electron Liquid Correlation Energies for Local Spin-Density Calculations: A Critical Analysis. Can. J. Phys. 1980, 58, 1200-1211.

5. $\quad$ Lee, C. T.; Yang, W. T.; Parr, R. G., Development of the Colle-Salvetti CorrelationEnergy Formula into a Functional of the Electron Density. Phys. Rev. B 1988, 37, 785-789.

6. Becke, A. D., Density-Functional Thermochemistry .Iii. The Role of Exact Exchange. J. Chem. Phys. 1993, 98, 5648-5652.

7. Stephens, P. J.; Devlin, F. J.; Chabalowski, C. F.; Frisch, M. J., Ab-Initio Calculation of Vibrational Absorption and Circular-Dichroism Spectra Using Density Functional Force Fields. J. Phys. Chem. 1994, 98, 11623-11627.

8. Ditchfield, R.; Hehre, W. J.; Pople, J. A., Self-Consistent Molecular-Orbital Methods. Ix. An Extended Gaussian-Type Basis for Molecular-Orbital Studies of Organic Molecules. $J$. Chem. Phys. 1971, 54, 724-728.

9. Valiev, M.; Bylaska, E. J.; Govind, N.; Kowalski, K.; Straatsma, T. P.; Van Dam, H. J. J.; Wang, D.; Nieplocha, J.; Apra, E.; Windus, T. L.; de Jong, W., Nwchem: A

Comprehensive and Scalable Open-Source Solution for Large Scale Molecular Simulations. Comput. Phys. Commun. 2010, 181, 1477-1489.

10. Phillips, J. C.; Braun, R.; Wang, W.; Gumbart, J.; Tajkhorshid, E.; Villa, E.; Chipot, C.; Skeel, R. D.; Kale, L.; Schulten, K., Scalable Molecular Dynamics with Namd. J. Comput. Chem. 2005, 26, 1781-1802.

11. Best, R. B.; Zhu, X.; Shim, J.; Lopes, P. E. M.; Mittal, J.; Feig, M.; MacKerell, A. D., Optimization of the Additive Charmm All-Atom Protein Force Field Targeting Improved Sampling of the Backbone $\vartheta, \Psi$ and Side-Chain X(1) and X(2) Dihedral Angles. J. Chem. Theory Comput. 2012, 8, 3257-3273.

12. Huang, J.; MacKerell Jr, A. D., Charmm36 All-Atom Additive Protein Force Field: Validation Based on Comparison to Nmr Data. J. Comput. Chem. 2013, 34, 2135-2145.

13. Hardy, D. J.; Wu, Z.; Phillips, J. C.; Stone, J. E.; Skeel, R. D.; Schulten, K., Multilevel Summation Method for Electrostatic Force Evaluation. J. Chem. Theory Comput. 2015, 11, 766-779.

14. Jorgensen, W. L.; Chandrasekhar, J.; Madura, J. D.; Impey, R. W.; Klein, M. L., Comparison of Simple Potential Functions for Simulating Liquid Water. J. Chem. Phys. 1983, 79, 926-935.

15. Ghadiri, M. R.; Granja, J. R.; Milligan, R. A.; Mcree, D. E.; Khazanovich, N., SelfAssembling Organic Nanotubes Based on a Cyclic Peptide Architecture. Nature 1993, 366, 324-327.

16. Migliore, A.; Polizzi, N. F.; Therien, M. J.; Beratan, D. N., Biochemistry and Theory of Proton-Coupled Electron Transfer. Chem. Rev. 2014, 114, 3381-3465.

17. Migliore, A.; Nitzan, A., Nonlinear Charge Transport in Redox Molecular Junctions: A Marcus Perspective. ACS Nano 2011, 5, 6669-6685. 
18. Migliore, A., Full-Electron Calculation of Effective Electronic Couplings and Excitation Energies of Charge Transfer States: Application to Hole Transfer in DNA ПStacks. J. Chem. Phys. 2009, 131, 114113.

19. Migliore, A., Nonorthogonality Problem and Effective Electronic Coupling Calculation: Application to Charge Transfer in Pi-Stacks Relevant to Biochemistry and Molecular Electronics. J. Chem. Theory Comput. 2011, 7, 1712-1725.

20. Zhao, Y.; Truhlar, D. G., The M06 Suite of Density Functionals for Main Group Thermochemistry, Thermochemical Kinetics, Noncovalent Interactions, Excited States, and Transition Elements: Two New Functionals and Systematic Testing of Four M06-Class Functionals and 12 Other Functionals. Theor Chem Acc 2008, 120, 215-241.

21. Zhao, Y.; Truhlar, D. G., Density Functional for Spectroscopy: No Long-Range SelfInteraction Error, Good Performance for Rydberg and Charge-Transfer States, and Better Performance on Average Than B3lyp for Ground States. J Phys Chem A 2006, 110, 1312613130.

22. Yanai, T.; Tew, D. P.; Handy, N. C., A New Hybrid Exchange-Correlation Functional Using the Coulomb-Attenuating Method (Cam-B3lyp). Chem. Phys. Lett. 2004, 393, 51-57. 